\title{
Análisis bibliométrico del concepto de resiliencia aplicado al desarrollo regional
}

\author{
Rudinei Kock Exterckoter \\ Instituto Federal Catarinense - Campus Concórdia \\ rudinei.exterckoter@ifc-concordia.edu.br
}

\section{Antoni Francesc Tulla Pujol}

Universitat Autònoma de Barcelona. Departament de Geografia

antoni.tulla@uab.cat

\section{Clécio Azevedo da Silva}

Universidade Federal de Santa Catarina

clecio@cfh.ufsc.br

\section{Resumen}

El concepto de resiliencia es mucho más conocido en las ingenierías y en las ciencias ecológicas, aunque, durante los últimos años, se utiliza cada vez más en los estudios sobre economía regional. Simultáneamente, se observa un esfuerzo de diversos investigadores para consolidar este concepto en el campo del desarrollo regional. La resiliencia de las regiones se relacionaría con la capacidad de las mismas de anticiparse y de prepararse para responder a escenarios negativos o de crisis. Sin embargo, aún no hay mucha información sobre la evolución de este concepto en las ciencias sociales. El presente artículo muestra los resultados del análisis bibliométrico llevado a cabo a partir de la literatura sobre la resiliencia aplicada al desarrollo regional. El estudio realizado permitió, entre otras cosas, profundizar en la comprensión de la evolución del uso del concepto de resiliencia, identificando a los autores y a las publicaciones que más han contribuido a su abordaje, así como las terminologías más usadas, los lugares y los años de las publicaciones más relevantes. Tales resultados demuestran que, a pesar de ser un concepto nuevo y en proceso de adopción por las ciencias sociales, ya figura como un instrumento importante para explicar las diferencias en la capacidad de adaptación económica de las regiones bajo contextos desfavorables.

Palabras clave: desarrollo regional; resiliencia; bibliometría; ciencias sociales. 
Resum. Anàlisi bibliomètrica del concepte de resiliència aplicat al desenvolupament regional

El concepte de resiliència és molt conegut a les enginyeries i a les ciències ecològiques, encara que, durant els darrers anys, s'utilitza cada vegada més en els estudis sobre economia regional. Simultàniament, s'observa un esforç de diferents investigadors per consolidar l'ús d'aquesta noció en el desenvolupament regional. Així, la resiliència de les regions estaria lligada a la capacitat que tenen d'anticipar-se als fets i de preparar-se per respondre a escenaris negatius o de crisi. Fins ara, encara hi ha poca informació sobre com ha evolucionat aquest concepte nou a les ciències socials. En aquest sentit, el present article mostra els resultats de l'anàlisi bibliomètrica de la literatura sobre la resiliència en l'àmbit del desenvolupament regional. Aquesta anàlisi va permetre, entre altres coses, aprofundir en la comprensió sobre l'evolució de l'ús del concepte de resiliència, identificant els autors i les revistes que van contribuir més a estudiar el tema, així com la terminologia més utilitzada, els llocs i els anys de publicació més importants. Aquests resultats demostren que, tot i ser un concepte nou i en procés d'adopció per part de les ciències socials, ja figura com un instrument important per explicar les diferències en la capacitat d'adaptació econòmica de les regions que pateixen situacions desfavorables.

Paraules clau: desenvolupament regional; resiliència; bibliometria; ciències socials.

Résumé. Analyse bibliométrique de la notion de résilience appliquée au développement régional

Le concept de résilience a été utilisé pour la première fois dans le domaine scientifique de l'ingénierie mécanique, puis il s'est étendu vers d'autres domaines de la science, comme l'écologie, la psychologie et, plus récemment les sciences sociales, tout particulièrement dans le domaine du développement régional. Bien qu'il soit considéré comme un concept multidisciplinaire, il présente des particularités en fonction du champ disciplinaire où il est employé. Ainsi, alors que dans l'écologie, la résilience décrit la capacité d'un écosystème à résister aux chocs extérieurs et à y répondre plutôt que de simplement dépérir et mourir, dans le domaine du développement régional la résilience est liée à la capacité des régions, des villes et des collectivités à prévoir, préparer, réagir et se remettre d'une rupture ou d'une crise. Dans ce domaine, apparaissent aussi des ouvrages exclusivement consacrés au traitement des systèmes ruraux sous la terminologie de " résilience du développement rurale ». Ce document contribue aux discussions en cours sur la résilience rurale. Il explore ainsi l'évolution conceptuelle de ce terme, avec l'émergence de l'expression « résilience du développement régional » dans la littérature et il identifie les défis conceptuels et méthodologiques de cette nouvelle approche. Les résultats démontrent que bien qu'il s'agisse d'un concept récent dans les sciences sociales et qui doive encore être approfondi, la résilience rurale ou la résilience du développement régional apportent beaucoup à la compréhension des stratégies d'adaptation adoptées par les agriculteurs de régions à situation défavorable.

Mots-clés: développement régional; résilience; bibliométrie; sciences sociales.

\section{Abstract. Bibliometric Analysis of the Concept of Resilience Applied to Regional Development}

Resilience is a much known concept in ecological sciences and engineering that has gained increased use in recent years in studies on the social sciences, and particularly on regional economics. Several researchers have attempted to consolidate the use of this concept in regional development by linking the resilience of regions to their capacity to anticipate and prepare for, respond to and recover from a disruption. However, there is little information about how this new concept has evolved together with the social sciences. This article presents the results of a bibliometric analysis of resilience in the context of regional develop- 
ment whose aim is, among other things, to deepen the understanding of how the use of the concept of resilience has evolved, and identify the authors and journals that contribute most to the study of this subject, as well as the most commonly used terminology and the locations and years of the most important publications. The results demonstrate that although resilience is a new concept that is in the process of being adapted to the social sciences, it has already become an important instrument in explaining differences in regions' capacity for economic adjustment in unfavorable scenarios.

Keywords: regional development; resilience; bibliometrics; social sciences.

\section{Sumario}

1. Introducción 4. Conclusiones

2. Estrategias metodológicas Agradecimientos

3. Análisis bibliométrico y discusiones Referencias bibliográficas

\section{Introducción}

En los últimos años, el uso de la noción de resiliencia se ha popularizado en el ámbito de los estudios regionales y territoriales (Méndez, 2012 y 2013), y se ha convertido en un nuevo concepto útil para apoyar la interpretación de fenómenos complejos que se desarrollan en los territorios (Pendall et al., 2009). Su uso se está generalizando para revelar cómo las regiones crecen, se desarrollan económicamente y responden a los procesos de crisis sin que se colapsen (Hopkins, 2008; Hudson, 2009; Pendall et al., 2009; Christopherson et al., 2010; Pike et al., 2010; Simmie y Martin, 2010; Méndez, 2012 y 2013; entre otros). Así, la resiliencia sería una medida teórica de la capacidad de un sistema socioecológico de absorber perturbaciones y reorganizarse, de modo que mantenga lo esencial de sus funciones, su estructura, su identidad y su retroalimentación (Hopkins, 2008; Hudson, 2009).

En los estudios regionales, el uso del concepto de resiliencia se basa, fundamentalmente, en dos aspectos. El primero lo vincula a la capacidad de adaptación de una región o territorio. En este sentido, dicha noción daría cuenta de un retorno a una trayectoria de equilibrio en el plan territorial, a través de adaptaciones de las estructuras vigentes (Simmie y Martin, 2010). Esta perspectiva deriva de una migración del concepto desde otros campos de las ciencias naturales. Por ejemplo, la resiliencia de los materiales o de los sistemas bióticos, que mide la capacidad de resistir a fuerzas externas y retornar rápidamente al equilibrio anterior, utiliza la idea de elasticidad o de flexibilidad para absorber y acomodar cambios sin experimentar una transformación estructural o sin riesgo de colapso.

La segunda perspectiva recurre a un planteamiento más ajustado al tema del desarrollo económico. Se aleja de la idea de retorno a una situación de 
equilibrio y enfatiza la capacidad de adaptación a los cambios dinámicos que tienen lugar en el territorio (Pendall et al., 2009; Christopherson et al., 2010; Pike et al., 2010; Simmie y Martin, 2010; Méndez, 2012; entre otros). Según esta postura, la resiliencia podría ser explorada para mejorar las condiciones de desarrollo de regiones, ciudades o comunidades, lo cual facilitaría la conexión con las tendencias evolutivas de tipo económico, social, ambiental o tecnológico. Asimismo, la promoción de la capacidad de adaptación del territorio en un contexto de incertidumbre podría representar una importante innovación en las estrategias de desarrollo regional.

Este segundo punto de vista se acerca a la economía institucional, al suponer que las empresas, las organizaciones y las instituciones están cambiando permanentemente y que sus conductas ayudan a configurar los ambientes económicos. En este sentido, considerar la resiliencia como la capacidad de adaptarse puede ser útil y orientador para entender cómo las regiones responden a las crisis y a las perturbaciones a lo largo de sus trayectorias de desarrollo.

Entre tanto, el debate va tomando forma justo ahora. Al entusiasmo de los que apuestan por el concepto de resiliencia, se contrapone la cautela de autores como Hudson (2009), que reconoce que el concepto empieza a situarse en el debate, pero también afirma que todavía hay bastante incertidumbre sobre las implicaciones de su uso. Hassink (2010) advierte que es necesario analizar la validez de la noción para el desarrollo regional, porque se trata, fundamentalmente, de un concepto de «moda». Por su parte, Pike et al. (2010) consideran que aún es prematuro tomarlo como una acepción útil para la teoría del desarrollo regional.

En medio de este debate, el presente artículo se propone revisar cómo se ha introducido el concepto de resiliencia en la literatura sobre el desarrollo regional. Para registrar y medir la configuración del debate y su evolución en los últimos años, hemos utilizado el análisis bibliométrico. Este nos ha posibilitado realizar inferencias a partir de una amplia base de datos disponibles. Esta técnica nos ha permitido ubicar dónde y cuándo se han introducido las principales publicaciones sobre la idea de resiliencia, así como caracterizar su evolución en el tiempo y en el espacio. Posteriormente, identificamos los principales autores que se han valido de esta idea, cuáles son las revistas que más han publicado artículos al respecto, en qué países el concepto despertó mayor interés y cuáles han sido los términos más adoptados, entre otras informaciones.

Este trabajo contará con dos secciones principales, además de la presente introducción y de las conclusiones. Mientras que la primera trata de los aspectos metodológicos involucrados en la recolección de datos, la segunda ofrecerá los resultados del análisis bibliométrico y su discusión.

\section{Estrategias metodológicas}

En este apartado, se distinguen dos etapas que orientaron la organización del análisis. La primera tiene que ver con el proceso de recolección de datos y la segunda, con la metodología desarrollada para llevar adelante el análisis bibliométrico. 


\subsection{Para la recolección de datos}

En una primera etapa, se leyeron diversos artículos que discutían la presencia del concepto de resiliencia en los estudios sobre desarrollo regional. Estas lecturas sirvieron como una primera aproximación al estado actual del debate. En seguida se buscaron los términos resilience y regional development en las bases de datos Scopus y Web of Science. De esta forma, se localizaron diversos trabajos. Mediante la lectura de sus títulos y resúmenes, se confirmó que se trataba de artículos de interés. El análisis de sus referencias bibliográficas permitió localizar otros artículos interesantes.

Tal estrategia nos permitió seleccionar siete expresiones principales con elevada frecuencia de uso, que pasaron a ser consideradas los términos de búsqueda de este trabajo (tabla 1).

La selección de las expresiones adoptadas por los autores fue una medida importante para limitar la investigación al ámbito del desarrollo regional, con lo cual se evitó la inclusión innecesaria de un gran número de trabajos de otras áreas científicas que suelen utilizar el concepto de resiliencia. No obstante, es importante destacar que la selección estuvo abierta a las más variadas formas de explorarlo, ya que la incorporación de la resiliencia en los estudios del desarrollo regional es muy reciente.

La opción de restringir los términos de búsqueda a la lengua inglesa se justifica, en primer lugar, porque casi la totalidad de las publicaciones referentes a la resiliencia se encuentra en este idioma, conforme a lo demostrado por Janssen et al. (2006), y, en segundo lugar, porque las bases bibliográficas más importantes usan el inglés para registrar las contribuciones científicas, aunque el idioma de origen del trabajo pueda ser otro.

Otra decisión metodológica importante fue la definición del período de búsqueda. Como la recolección de datos se realizó entre 2014 e inicios de 2015, se optó por limitar la búsqueda a las contribuciones publicadas antes del 31 de diciembre de 2014, lo cual posibilita una mejor caracterización

Tabla 1. Términos de búsqueda elegidos para la revisión de los estudios sobre la resiliencia en el desarrollo regional ${ }^{*}$

\begin{tabular}{ll}
\hline Términos de búsqueda & Referencias \\
\hline Regional resilience & Hill et al. (2008); Hopkins (2008); Simmie y Martin (2010); Christopherson \\
& et al. (2010); Bristow (2010) \\
Community resilience & King (2008); Magis (2010) \\
Resilient regions & Hudson (2009); Bristow (2010) \\
Resilient cities & Bristow (2010); Méndez (2012 y 2013) \\
Rural resilience & Franklin et al. (2011); Glover (2012) \\
Social resilience & Adger (2000) \\
Urban resilience & Campanella (2006); Polèse (2010); Méndez (2012 y 2013)
\end{tabular}

Fuente: elaboración propia.

* Términos de búsqueda en castellano: resiliencia regional, resiliencia comunitaria, regiones resilientes, ciudades resilientes, resiliencia rural, resiliencia social y resiliencia urbana. 
evolutiva de este concepto en los estudios sobre desarrollo regional. En lo que concierne a las bases bibliográficas, se decidió recurrir a dos fuentes tradicionales de investigación científica que presentan un importante acervo en el área de las ciencias sociales: Scopus y Web of Science. En Scopus, la búsqueda fue restringida a la gran área denominada "Social Sciences \& Humanities» ("Ciencias Sociales y Humanidades»). Análogamente, en la Web of Science, la búsqueda se limitó a la gran área denominada "Social Sciences» («Ciencias Sociales»). En ambas bases, se identificaron documentos con formato de artículo (journal article), para luego identificar, en cada trabajo, el título (title), el resumen (abstract) y las palabras clave (keywords). Adicionalmente, se limitaron las áreas de búsqueda en cada base. Además, en cada una, los términos de búsqueda social resilience ('resiliencia social') y community resilience ('resiliencia comunitaria') fueron investigados junto al término development ('desarrollo'). Es importante destacar que restringir la búsqueda a una gran área en cada base de datos e incluir la palabra development junto a los términos anteriormente mencionados permitieron reducir el número de artículos no deseados. Esta decisión se basó en pruebas que demostraron que, si no se hubiesen tenido en cuenta estas restricciones, se habría incluido un gran número de trabajos que trataban sobre la resiliencia en las áreas de la salud o la ecología, y que no eran de interés para esta investigación. Con estos criterios, se llevó a cabo el análisis bibliométrico.

\subsection{Metodología usada en el análisis bibliométrico}

A partir del total de trabajos identificados, se realizaron diferentes análisis estadísticos con la finalidad de caracterizar el comportamiento de la variable en las bases bibliográficas (Scopus y Web of Science). Posteriormente, con el uso del programa EndNote ${ }^{\circledR}$, los datos fueron filtrados y los duplicados, eliminados. Los artículos fueron revisados uno a uno para que pudiéramos establecer las correlaciones correctas con el área del desarrollo regional.

Para facilitar la ejecución de esta etapa, se adoptaron distintos «dominios lingüísticos» de clasificación de los artículos. Esta tarea no es nada fácil, ya que, según afirma Santos (2009), tales dominios interactúan y no son estáticos. Este mismo autor propone la adopción de los siguientes cuatro dominios: a) Ambiente, ecosistema y recursos naturales; $b$ ) Catástrofes naturales y desastres humanos; $c$ ) Organizaciones, empresas y economía, y $d$ ) Territorios y procesos de desarrollo. Con la intención de mejorar la representatividad de los resultados, se incluyeron los dominios $e$ ) Pueblos tradicionales y medio ambiente, y $f$ ) Otros. La inclusión de los trabajos en cada uno de estos dominios se hizo a partir del título y el resumen, y se consultaba el resto del artículo cuando se presentaba alguna duda.

Finalmente, se decidió concentrar el análisis en el dominio «Territorios y procesos de desarrollo», ya que este se ligaba más directamente a los objetivos de dicho trabajo. 


\section{Análisis bibliométrico y discusiones}

Este apartado se divide en dos partes. En la primera, se clasifican los artículos según país de origen, lengua, dominio, palabras clave, año y lugar de procedencia. En la segunda, se analizan los artículos más relevantes correspondientes al dominio «Territorios y procesos de desarrollo».

\subsection{Estadisticas generales y depuración de los datos}

La búsqueda permitió la localización de 426 artículos, 222 obtenidos de la base Scopus y 204 de la base Web of Science. La exploración de este conjunto permitió otras informaciones complementarias, tales como qué autores y qué revistas contribuyen más al estudio del tema, así como los lugares y los años con un mayor número de artículos publicados.

Como es posible observar en la figura 1 , de los siete términos de búsqueda, destacaron cinco (community resilience, urban resilience, regional resilience, resilient cities y social resilience), ordenados respectivamente en función del número de artículos encontrados.

Por otra parte, los términos resilient regions y rural resilience solo aparecieron mencionados en un pequeño número de artículos. Sin embargo, su presencia en la base de datos se justifica en función de la calidad de los mismos. Además de ser útiles para la investigación, cabe destacar que ellos no coincidieron con ninguno de los otros términos de búsqueda. También es importante puntualizar que, aunque con Scopus se han podido ubicar más trabajos que con Web of Science, no se puede afirmar todavía cual fue la base más adecuada, ya

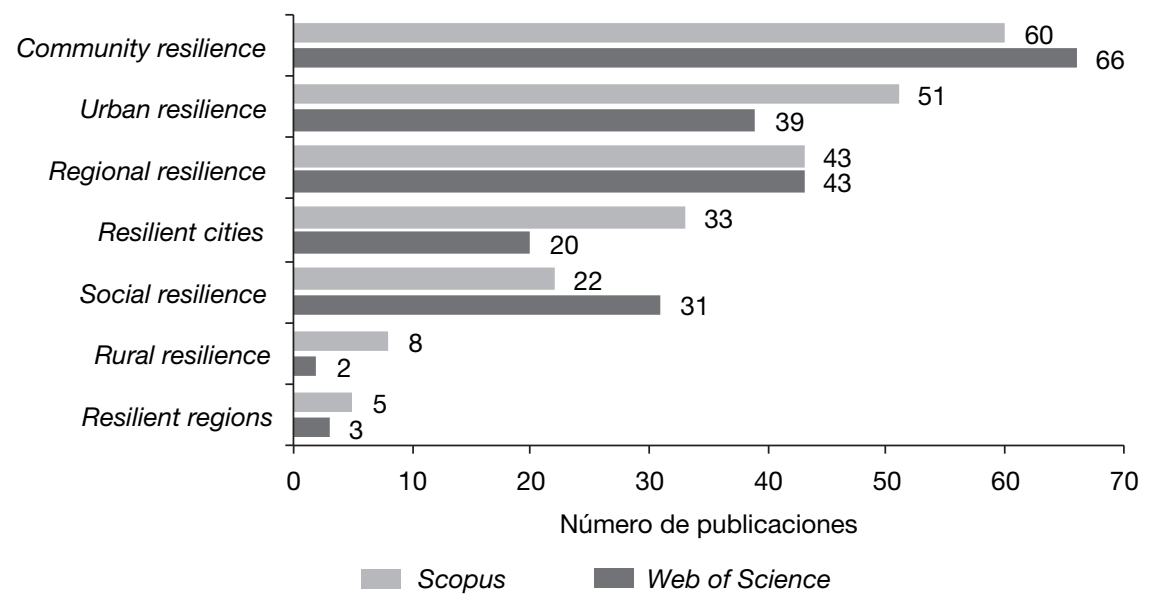

Figura 1. Número de artículos para cada término de búsqueda en las bases de investigación Scopus y Web of Science.

Fuente: elaboración propia. 


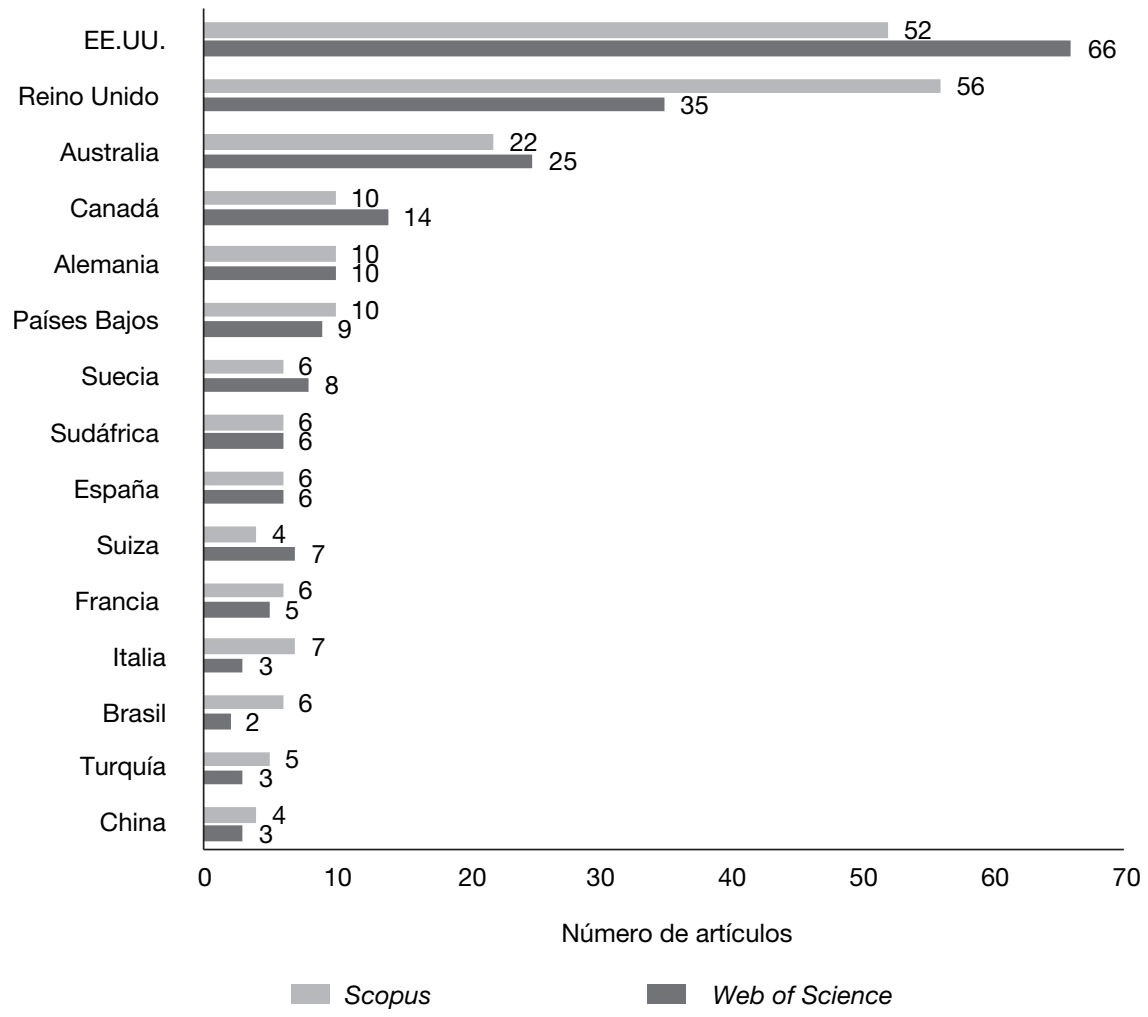

Figura 2. Identificación de los 15 países con mayor número de artículos publicados en sus revistas, considerando todos los términos de búsqueda en Scopus y Web of Science.

Fuente: elaboración propia.

que el total de trabajos obtenidos tras la eliminación de los duplicados supera el número encontrado en cada base, como veremos más adelante.

Tomando como referencia la información de las bases de datos, en las figuras 2 y 3 se han podido identificar los países y las lenguas en que fueron publicados los trabajos analizados.

Las figuras 2 y 3 evidencian el predominio de trabajos producidos en los Estados Unidos y el Reino Unido como centros de publicación, así como el uso casi exclusivo de la lengua inglesa para difundirlos. Estos resultados coinciden con los de Janssen et al. (2006), quienes también observaron que las publicaciones sobre resiliencia (en este caso, de un modo más general para diferentes áreas) adquirían preeminencia en estos mismos países y, en su mayoría, se expresaban en dicha lengua. Como consecuencia de ello, las revistas de dichos países tienden a concentrar las publicaciones más relevantes sobre resiliencia. Aparentemente, este predominio conduce a que la divulgación de los trabajos 


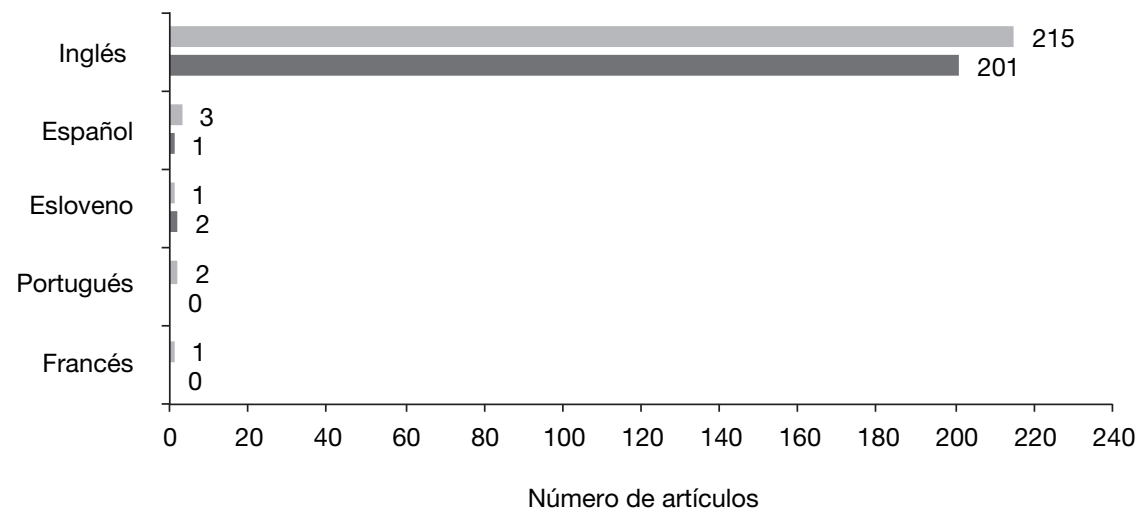

Scopus

Web of Science

Figura 3. Número de artículos publicados según la lengua, considerando todos los términos de búsqueda en Scopus y Web of Science.

Fuente: elaboración propia.

sobre la temática en otras partes del mundo sea más atractiva en la actualidad, pues abre caminos de investigación en otros países.

Los datos presentados ofrecen indicios importantes para avanzar en el estudio de la resiliencia. Entre tanto, para proseguir con el análisis bibliométrico, fue necesario que los resultados obtenidos en las dos bases de datos fuesen depurados para identificar y eliminar los artículos duplicados. Para ejecutar esta tarea, fue utilizado el programa EndNote®. A través de él, se constató la presencia de 121 duplicaciones. Al final, quedaron 305 artículos, los cuales pasaron a ser analizados individualmente. Este análisis tenía por objetivo clasificar los artículos en diferentes dominios, de acuerdo con el objetivo de documentar la resiliencia en el área de desarrollo regional. Como ya se ha mostrado en la metodología, los dominios utilizados fueron adaptados a los propuestos por Santos (2009), añadiendo dos nuevos: «Pueblos tradicionales y medio ambiente» y «Otros» (figura 4). Para clasificar cada artículo en un dominio, se realizó la lectura del título, así como del resumen, y, en los casos que generaban dudas, la lectura se extendió al resto del trabajo.

Es importante resaltar que los seis dominios usados para agrupar los diferentes artículos se interrelacionan y no son estancos. Todos los textos abordan el tema de la resiliencia, aunque 138 se focalizan en el proceso de desarrollo de las regiones a partir de la perspectiva de la resiliencia. De esta forma, dichos artículos son los que despiertan el mayor interés para este trabajo y los que fueron estudiados más minuciosamente. Tal resultado confirma la importancia de estudiar la resiliencia en el ámbito del desarrollo regional y territorial ( $45,2 \%$ del total). 


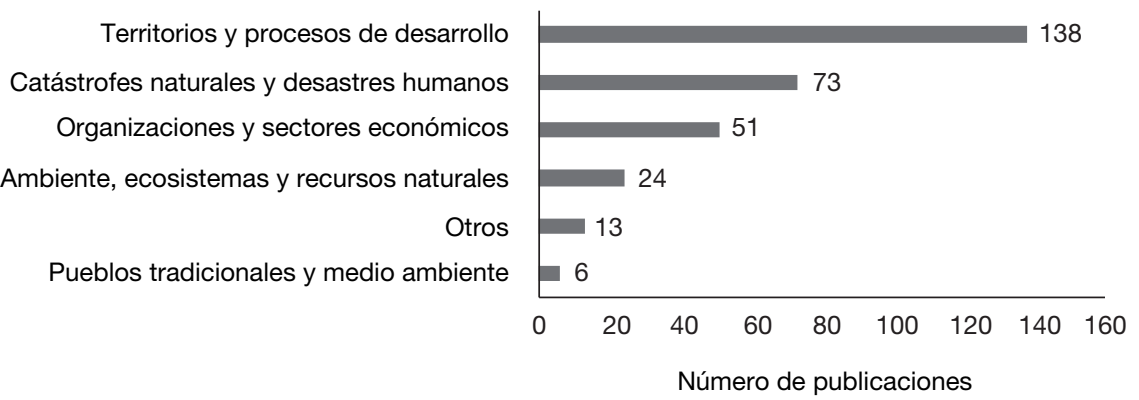

Figura 4. Número de artículos sobre resiliencia por cada dominio lingüístico estudiado.*

Fuente: elaboración propia.

* Dominios lingüísticos en inglés: Territories and development processes, Natural catastrophes and human disasters, Economic sectors and organizations, Environment, Ecosystems and natural resources, Others y Traditional communities and environment.

Cabe destacar también que el dominio «Otros» fue creado para agrupar los artículos, que, aunque localizados a través de la estrategia metodológica anteriormente presentada, no se encuadraban en ninguno de los dominios propuestos. En general, estos estaban relacionados con la resiliencia en el área de la salud o de la psicología. Situaciones como esta pueden plantear errores sistemáticos en la selección de los términos de búsqueda usados en la investigación. Sin embargo, este no es el caso para el presente trabajo, ya que apenas 13 artículos de un total de 305 no correspondieron al tema central, a pesar de tratarse de la resiliencia. Esto ocurrió, especialmente, por la forma como se registraron en las bases de datos, en las que se incluyen palabras clave que no representan el artículo. Esta estrategia es utilizada por algunos autores para ampliar las posibilidades de que sus trabajos sean localizados en investigaciones de áreas distintas.

Otro dato que llama la atención es el aumento del interés por la temática a lo largo del tiempo, como se puede observar en la figura 5. En ella, se presenta la distribución temporal del total de trabajos correspondientes al dominio "Territorios y procesos de desarrollo», el más relevante para esta investigación, en relación con el número total de artículos (305).

Se hace evidente que la exploración del concepto de resiliencia en artículos sobre el desarrollo regional se incrementó fuertemente en la última década. Esto caracteriza la difusión de un planteamiento teórico en pleno proceso de construcción. Los datos contenidos en la figura 6 ayudan a entender mejor este escenario, en la medida en que presenta la evolución de los términos buscados en los artículos que componen el dominio de interés.

Esta figura permite analizar la evolución temporal de cada término de búsqueda. Llama la atención la importancia que el término regional resilience presentaba en la primera fase de expansión de los estudios sobre resiliencia en el desarrollo regional, entre 2008 y 2010 . No obstante, a partir de 2011, el 


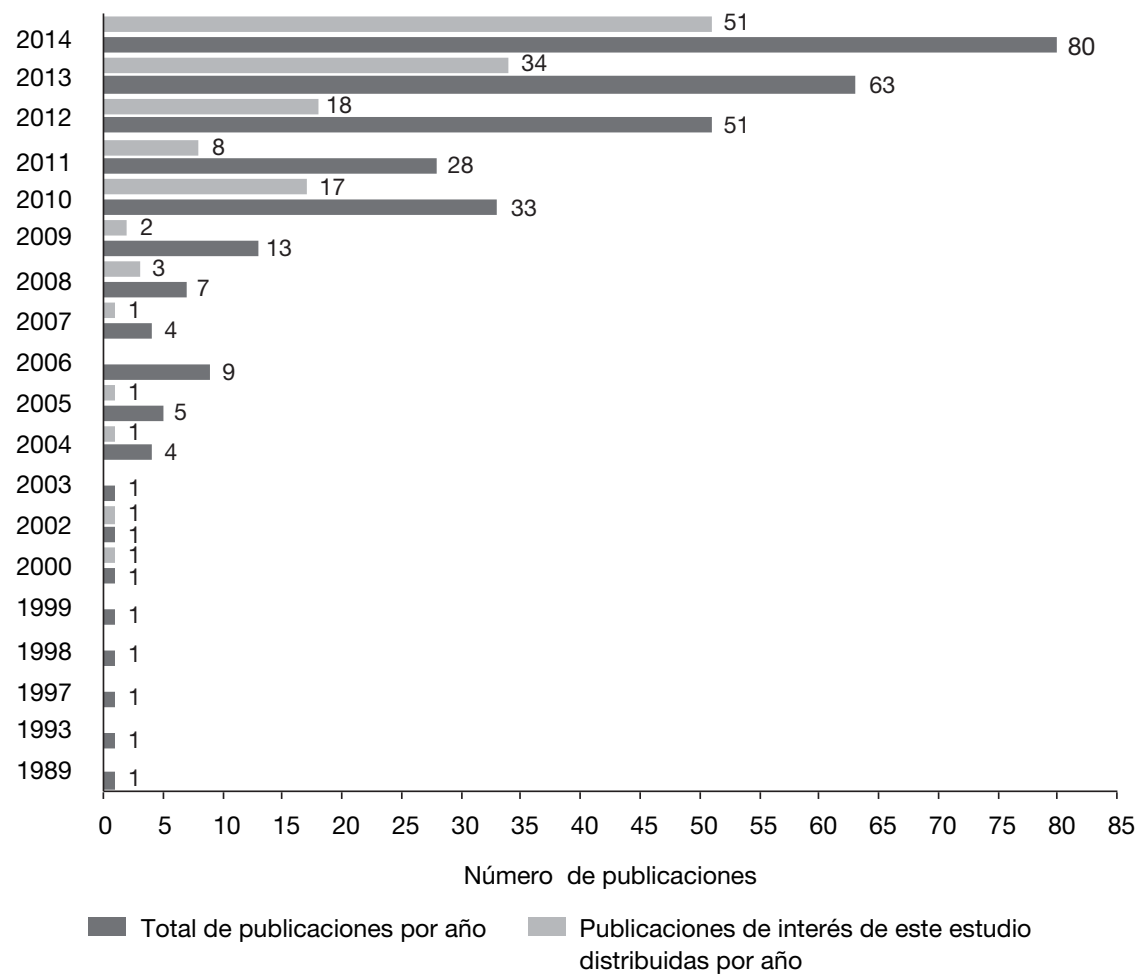

Figura 5. Evolución temporal del número de artículos del dominio «Territorios y procesos de desarrollo".

Fuente: elaboración propia.

término regional resilience comenzó a ceder espacio a community resilience (que sí aparece ya como importante desde 2008), resilient cities y urban resilience, que vuelven a ser más ampliamente utilizados en 2014. En realidad, en las lecturas de los artículos más recientes, se observa que es cada vez más común el uso del término resilience y, por lo tanto, la aplicación del mismo al sistema de estudio, como, por ejemplo: community resilience, resilient cities y urban resilience. Este proceso describe una especie de fraccionamiento del tema de estudio, implicando cambios o reconfiguraciones del objeto a ser investigado. De hecho, el interés pasa a concentrarse, cada vez más, en sistemas más pequeños, como ciudades y/o comunidades. Cabe observar, además, la aparición reciente del término rural resilience, que también puede estar relacionado con la fragmentación señalada. En efecto, el concepto de resiliencia da las primeras señales de ser utilizado en los estudios sobre el desarrollo rural a principios de la década de 2010 (Glover, 2012; Wilson et al., 2013; Paniagua, 2013; Scott, 2013; Darnhofer, 2014). 


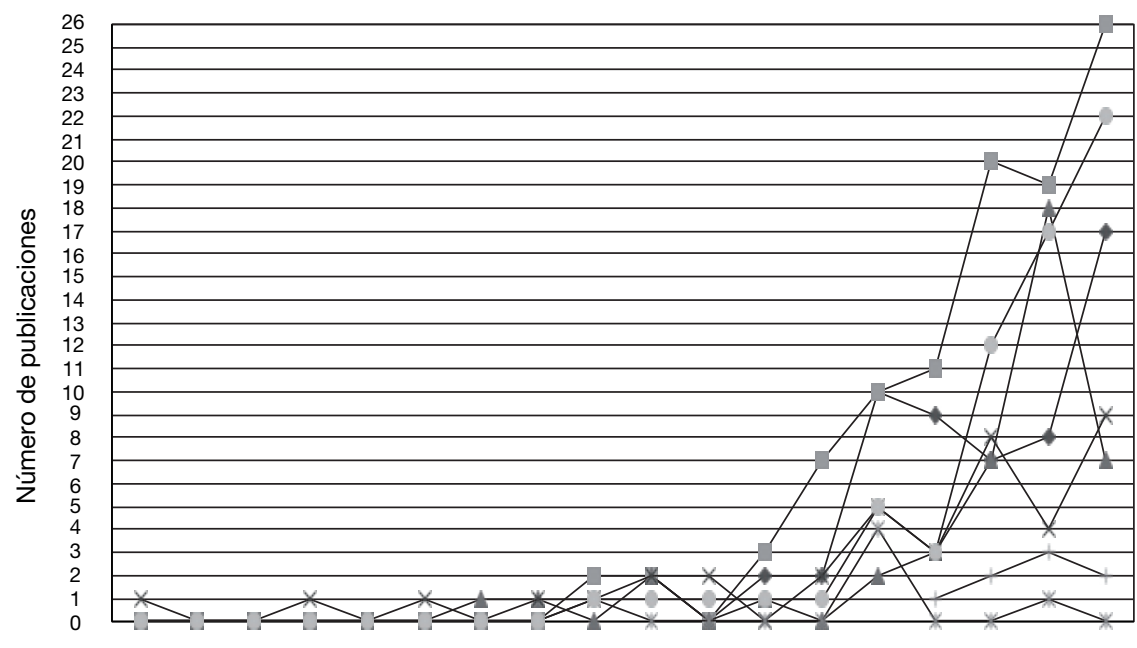

199719981999200020012002200320042005200620072008200920102011201220132014

Años

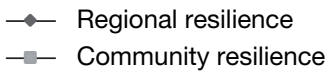

$\rightarrow-$ Social resilience
$-\leftarrow$ Resilient cities

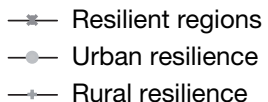

Figura 6. Evolución temporal del número de artículos del dominio «Territorios y procesos de desarrollo» según los términos de búsqueda seleccionados.

Fuente: elaboración propia.

Sin embargo, se constata la existencia de un mayor número de trabajos sobre resiliencia en áreas urbanas, en comparación con los referidos a las áreas rurales.

Siguiendo con el análisis detallado del dominio «Territorios y procesos de desarrollo», presentamos, en la figura 7, los documentos distribuidos según las revistas con más publicaciones. El conjunto de 138 artículos de ese dominio se encuentra distribuido en 81 revistas. Solo 26 de las cuales presentaron más de una publicación sobre la temática, como se muestra en la figura 7 .

Aunque los resultados presentes en la figura 7 demuestran la existencia de una gran dispersión de los artículos en un número elevado de revistas, se destaca la titulada Cambridge Journal of Regions Economy and Society, con once (11) artículos en total. De hecho, se trata de la revista inglesa con un mayor número de artículos publicados. Esto se debe, principalmente, a la edición de un volumen especial (número 3 de 2010) dedicado a explorar diferentes enfoques en relación con la resiliencia. Según el Consejo Editorial de la misma, la decisión de publicar esta edición especial fue motivada tanto por la popularidad que el término alcanzó, como por la necesidad de avanzar en la comprensión 


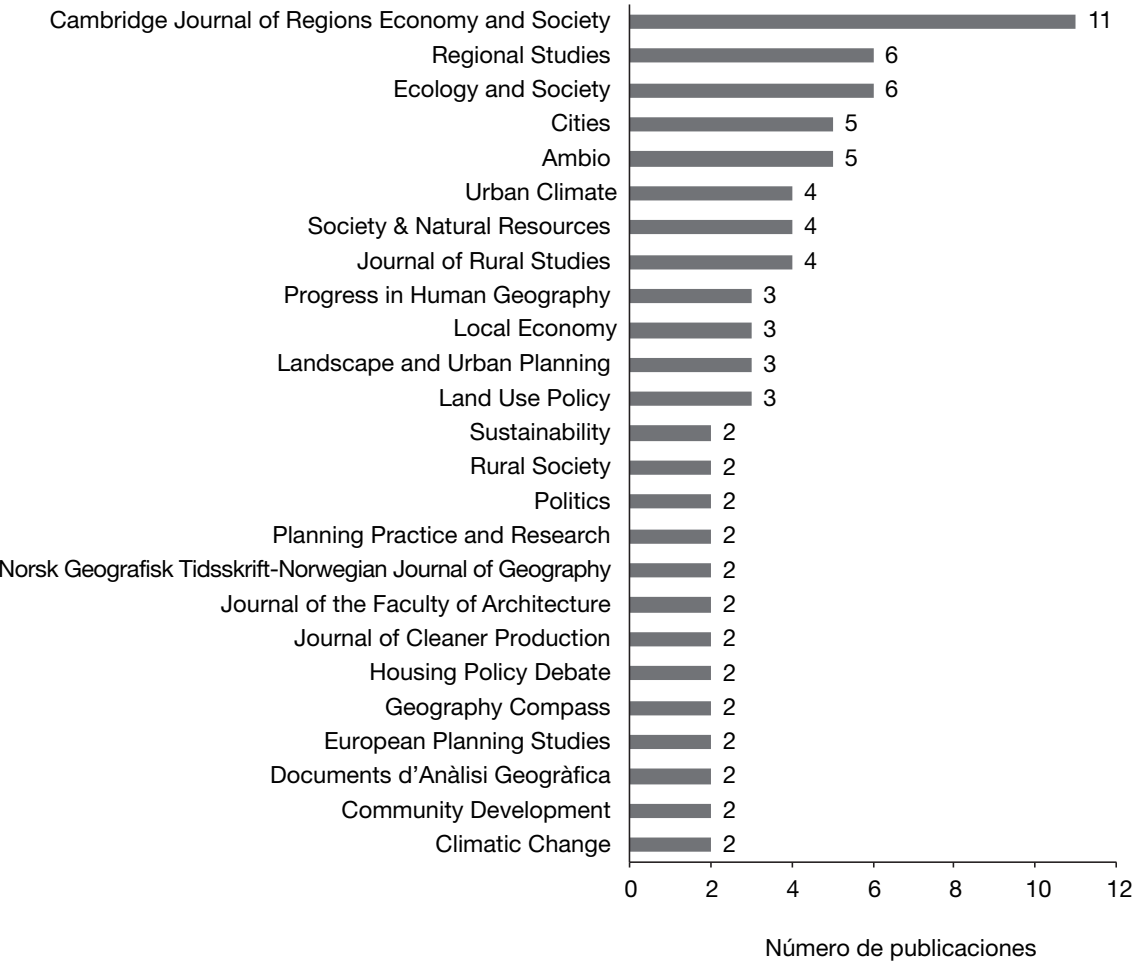

Figura 7. Revistas en que se publicaron los artículos utilizados en esta investigación.

Fuente: elaboración propia.

de las contribuciones del mismo en el estudio del proceso de desarrollo de las regiones. Esta edición especial también ayuda a explicar el mayor número de artículos sobre resiliencia en el año 2010 en relación con el año 2011 (figura 5).

Otro análisis relevante deriva del reconocimiento de las palabras clave más usadas por los artículos incorporados en el dominio "Territorios y procesos de desarrollo». Entre los trabajos de este dominio, se encontraron un total de 1014 palabras y/o expresiones clave, las cuales fueron analizadas de dos maneras. La primera forma de análisis se vincula con la identificación de las palabras clave que aparecieron más de 10 veces, sea de forma aislada y/o componiendo una expresión, y que se detallan en la tabla 2.

La palabra resilience es la que más aparece (117 veces), duplicando el uso del término urban (51 veces). También vale la pena destacar que, en 46 ocasiones, la palabra resilience es usada sin complementos. Ello refuerza la tendencia observada anteriormente en este trabajo, según la cual el uso de este término está aumentando. Otra situación ya discutida y que se confirma a través del 
Tabla 2. Listado de palabras clave usadas de forma aislada y/o en una expresión

\begin{tabular}{|c|c|c|c|}
\hline \multirow[b]{2}{*}{ Palabras clave } & \multicolumn{2}{|r|}{ Formas de uso* } & \multirow[b]{2}{*}{ Total } \\
\hline & Aislado & Componiendo una expresión & \\
\hline Resilience & 46 & 71 & 117 \\
\hline Urban & 0 & 51 & 51 \\
\hline Community & 7 & 32 & 39 \\
\hline Social & 1 & 36 & 37 \\
\hline System & 3 & 32 & 35 \\
\hline Development & 1 & 33 & 34 \\
\hline Economic & 0 & 33 & 33 \\
\hline Ecological & 0 & 27 & 27 \\
\hline Regional & 0 & 27 & 27 \\
\hline Change & 0 & 26 & 26 \\
\hline Adaptation & 18 & 7 & 25 \\
\hline Climate & 0 & 25 & 25 \\
\hline Economic & 0 & 22 & 22 \\
\hline Rural & 4 & 18 & 22 \\
\hline Cities & 6 & 15 & 21 \\
\hline Policy & 4 & 17 & 21 \\
\hline Planning & 5 & 14 & 19 \\
\hline Adaptive & 0 & 18 & 18 \\
\hline Environment & 1 & 16 & 17 \\
\hline Governance & 10 & 7 & 17 \\
\hline Vulnerability & 17 & 0 & 17 \\
\hline Sustainability & 13 & 3 & 16 \\
\hline Resource & 1 & 13 & 14 \\
\hline Disaster & 5 & 8 & 13 \\
\hline Sustainable & 0 & 12 & 12 \\
\hline Ecosystem & 1 & 11 & 12 \\
\hline Environmental & 0 & 12 & 12 \\
\hline Innovation & 5 & 7 & 12 \\
\hline Capacity & 5 & 6 & 11 \\
\hline Geography & 2 & 9 & 11 \\
\hline Innovation & 5 & 6 & 11 \\
\hline Regions & 8 & 3 & 11 \\
\hline
\end{tabular}

* Cabe destacar que se incluyeron solamente las palabras que aparecieron como mínimo diez veces, directamente o en expresión.

Fuente: elaboración propia.

reconocimiento de las palabras clave es el mayor número de artículos dedicados al estudio del espacio urbano (urban, cities), en detrimento de los destinados a las regiones (regional, regions) o a los espacios rurales (rural).

Una segunda forma de análisis fue dedicada a identificar solamente las expresiones que más veces aparecieron en los trabajos como palabras clave (tabla 3). 
Tabla 3. Expresiones más usadas como palabras clave en los artículos del dominio «Territorios y procesos de desarrollo"

\begin{tabular}{lc}
\hline Expresiones más usadas* $^{\star}$ & Total \\
\hline Community resilience & 19 \\
Climate change & 16 \\
Regional resilience & 12 \\
Urban resilience & 12 \\
Social-ecological systems & 9 \\
Sustainable development & 7 \\
Urban planning & 7 \\
Ecosystem services & 6 \\
Adaptive capacity & 5 \\
Ecological resilience & 5 \\
Evolutionary economic geography & 5 \\
Path dependence & 5 \\
Resilient cities & 5 \\
\hline
\end{tabular}

* Fueron incluidas solamente las expresiones que aparecían por lo menos cinco veces.

Fuente: elaboración propia.

Llama la atención que la segunda expresión más usada como palabra clave sea climate change. Los cambios climáticos son una preocupación recurrente en los trabajos que debaten la resiliencia. Podemos evaluar dicho interés desde dos puntos de vista: como un efecto inmediato de la migración del concepto desde las ciencias naturales, y que se diluirá a lo largo del tiempo, o, más bien, como expresión de un interés por incluir la cuestión climática en los estudios sobre el desarrollo.

Otro punto a destacar es el uso difundido en los trabajos de las expresiones community resilience, urban resilience y regional resilience. En particular, las dos primeras confirman la tendencia de fraccionamiento de la escala de estudio. Este fraccionamiento, muy probablemente, está relacionado con los problemas metodológicos a los que se enfrenta a la hora de medir la resiliencia (Darnhofer et al., 2010; Cabell y Oelofse, 2012; Paniagua, 2013). Tales dificultades son tanto más grandes y complejas cuanto más amplio es el sistema o la realidad que se estudia. En la evaluación de Darnhofer (2014), medir la resistencia en sistemas socioecológicos es "como mirar un blanco en movimiento".

La figura 8 distingue los artículos por el país de origen del primer autor.

Sobre un total de 138 artículos, 61 de los primeros autores de los trabajos realizan su investigación en Estados Unidos y en el Reino Unido. Solamente tres textos no fueron publicados en inglés. Finalmente, considerando las universidades de origen de los investigadores, se destacan la de Queensland, con 5 artículos; la de East Anglia, con 4, y la de Estocolmo, con 3. La Universidad de Queensland es la que cuenta con más textos producidos acerca de la resiliencia en Australia, con lo que se convierte en una referencia sobre el tema en este país. Cabe destacar también que C. Folke y N. Adger, con trabajos seminales 


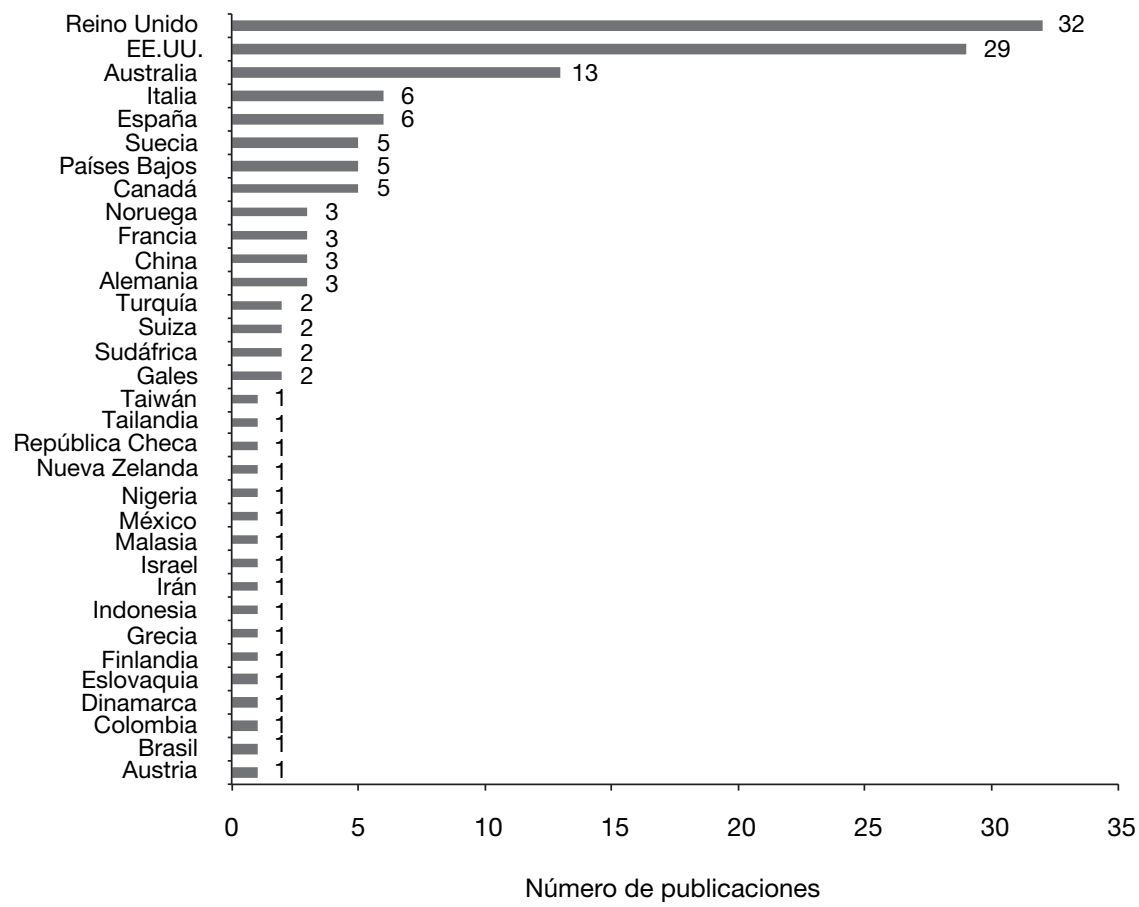

Figura 8. Distribución de los artículos del dominio «Territorios y procesos de desarrollo» por nacionalidad del primer autor.

Fuente: elaboración propia.

en la temática, pertenecen a las universidades de East Anglia (Reino Unido) y de Estocolmo (Suecia) ${ }^{1}$.

\subsection{Análisis de los artículos más relevantes del dominio "Territorios y procesos de desarrollo"}

Al avanzar en el análisis de los artículos, se verificó que los 138 trabajos de este dominio correspondían a 306 autores diferentes, aunque solo 17 de estos autores han producido más de un artículo. Quienes produjeron más estudios fueron G. Bristow y J. Ahern, con tres artículos cada uno. Aparentemente, este resultado responde al hecho de que la resiliencia hace poco que se incorporó al análisis regional, con lo que se reduce la posibilidad de que existan muchos autores con un gran número de publicaciones.

1. C. Folke ejerce como profesor de la Universidad de Estocolmo, mientras que N. Adger obtuvo el título de doctor y realizó sus estudios posdoctorales en la Universidad de East Anglia. 
Evidentemente, se podría localizar a los autores y a los artículos referentes a este tema desde otras formas. En nuestro caso, recurrimos a las bases de datos donde fue posible identificar los trabajos y, consecuentemente, los autores con textos con mayor número de citas (tabla 4). Teniendo en cuenta que un autor puede tener publicaciones sobre diversos temas, este análisis nos permitió identificar el número de citas del autor en relación con cada trabajo específico, en lugar de hacerlo respecto a su producción general. De esta forma, pudimos ubicar a los autores y a los artículos que se han convertido en referencia básica para los trabajos que discuten la resiliencia en el desarrollo regional.

Es importante también resaltar que estos datos corresponden a un espacio de tiempo corto y que, además, pueden variar en el futuro Así, por ejemplo, pueden identificarse artículos recientes de gran calidad, pero que todavía no alcanzaron un gran número de citas, debido al escaso tiempo en que han estado disponibles para su consulta por parte de otros investigadores.

Antes de avanzar en la discusión en torno a los autores y a los artículos más relevantes, es necesario destacar la importancia que ha adquirido la revista Cambridge Journal of Regions Economy and Society en la temática (tabla 4). Entre los 17 trabajos más relevantes de las bases Scopus y Web of Science, 5 fueron publicados en esta revista, lo que reafirma su posición destacada, conforme a lo señalado en la figura 7. También llama la atención el hecho de que los 17 artículos más citados se repiten en ambas bases, con algún cambio en el orden, lo que ratifica el uso de ambas bases de datos en nuestra investigación.

Los dos artículos más citados son los mismos en ambas bases: se trata de los trabajos de Adger (2000) y Norris et al. (2008). Estos presentan un número de citas muy superior a los artículos que aparecen en el tercer puesto de cada base. Así, mientras que Adger (2000) cuenta con un promedio de cinco veces más de citas, el de Norris et al. (2008) fue citado tres veces más que el trabajo que aparece en la tercera posición. Si bien, en el caso de Adger, el mayor número de citas se debe en parte a la antigüedad de la publicación, un análisis más detallado de ambos trabajos y del perfil de sus primeros autores puede ayudar a entender mejor estas cifras.

Neil Adger, profesor en Geografía de la Facultad de la Vida y Ciencias Ambientales de la Universidad de Exeter, en el Reino Unido, es miembro de la Resilience Alliance, red global de científicos que trabajan sobre la teoría y la práctica de la resiliencia de los sistemas socioecológicos para la sustentabilidad. Se ha dedicado a investigar los procesos de adaptación y resiliencia en el contexto de las transformaciones ambientales globales. Además, el artículo en cuestión («Social and ecological resilience: Are they related?») es el que introduce el concepto de resiliencia social. En este trabajo, el autor considera que dicha idea no solo permite describir la situación social y económica de las comunidades, sino también explorar los vínculos potenciales entre las denominadas resiliencia social y resiliencia ecológica. Desde entonces, el texto se convirtió en una referencia bibliográfica casi obligatoria para los trabajos que tratan la resiliencia en el desarrollo regional. 
Tabla 4. Artículos sobre resiliencia disponibles en Scopus y Web of Science, ordenados en función del número de veces que son citados en otros artículos

\begin{tabular}{|c|c|c|}
\hline \multicolumn{3}{|c|}{ Scopus } \\
\hline Referencias* & Revistas & N. ${ }^{\circ}$ de citas \\
\hline Adger (2000) & Progress in Human Geography & 644 \\
\hline Norris et al. (2008) & American Journal of Community Psychology & 449 \\
\hline Pickett et al. (2004) & Landscape and Urban Planning & 134 \\
\hline Simmie y Martin (2010) & Cambridge Journal of Regions Economy and Society & 118 \\
\hline Adger et al. (2002) & Ambio & 99 \\
\hline Magis (2010) & Society \& Natural Resources & 70 \\
\hline Walker et al. (2009) & Ecology and Society & 69 \\
\hline Ernstson et al. (2010) & Ambio & 65 \\
\hline Christopherson et al. (2010) & Cambridge Journal of Regions Economy and Society & 58 \\
\hline Bristow (2010) & Cambridge Journal of Regions Economy and Society & 52 \\
\hline Wilson (2010) & Transactions of the Institute of British Geographers & 50 \\
\hline Marshall et al. (2007) & Rural Sociology & 49 \\
\hline Hudson (2009) & Cambridge Journal of Regions Economy and Society & 49 \\
\hline Hassink (2010) & Cambridge Journal of Regions Economy and Society & 46 \\
\hline Berkes y Ross (2013) & Society \& Natural Resources & 45 \\
\hline Cote y Nightingale (2012) & Progress in Human Geography & 45 \\
\hline Wardekker et al. (2010) & Technological Forecasting and Social Change & 40 \\
\hline \multicolumn{3}{|c|}{ Web of Science } \\
\hline Referencias* & Revistas & N. ${ }^{\circ}$ de citas \\
\hline Adger (2000) & Progress in Human Geography & 460 \\
\hline Norris et al. (2008) & American Journal of Community Psychology & 320 \\
\hline Pickett et al. (2004) & Landscape and Urban Planning & 96 \\
\hline Adger et al. (2002) & Ambio & 92 \\
\hline Simmie y Martin (2010) & Cambridge Journal of Regions Economy and Society & 87 \\
\hline Walker et al. (2009) & Ecology and Society & 51 \\
\hline Christopherson et al. (2010) & Cambridge Journal of Regions Economy and Society & 48 \\
\hline Marshall et al. (2007) & Rural Sociology & 46 \\
\hline Ernstson et al. (2010) & Ambio & 44 \\
\hline Magis (2010) & Society \& Natural Resources & 44 \\
\hline Wardekker et al. (2010) & Technological Forecasting and Social Change & 39 \\
\hline Hassink (2010) & Cambridge Journal of Regions Economy and Society & 36 \\
\hline Berkes y Ross (2013) & Society \& Natural Resources & 35 \\
\hline Cote y Nightingale (2012) & Progress in Human Geography & 34 \\
\hline Bristow (2010) & Cambridge Journal of Regions Economy and Society & 33 \\
\hline Hudson (2009) & Cambridge Journal of Regions Economy and Society & 30 \\
\hline Wilson (2010) & Transactions of the Institute of British Geographers & 30 \\
\hline
\end{tabular}


Por su parte, Fran H. Norris actúa como profesora de la Escuela de Medicina Geisel en Dartmouth (EE. UU.), en el Área de Psiquiatría, Medicina Familiar y Comunidad. Norris ha trabajado fundamentalmente con la resiliencia comunitaria y la resiliencia social enfocada hacia problemáticas asociadas a desastres naturales y al terrorismo. El trabajo en cuestión tiene como título «Community Resilience as a Metaphor, Theory, Set of Capacities, and Strategy for Disaster Readiness" y se dedica a discutir el concepto de resiliencia aplicado a comunidades (community resilience). En este mismo trabajo, la autora recorre el camino evolutivo de dicho concepto en las diferentes ciencias, con el fin de argumentar y defender la importancia de la capacidad adaptativa de las comunidades en las respuestas a las diferentes crisis. El texto ha sido utilizado como base metodológica para trabajos sobre resiliencia en la psicología, en el tratamiento de la resiliencia comunitaria y también en el desarrollo regional. El amplio uso de este artículo en diferentes campos justificaría el gran número de citas.

La tabla 5 identifica los principales autores que publican textos sobre resiliencia y que fueron incluidos en el dominio «Territorio y procesos del desarrollo». A su vez, presenta las referencias bibliográficas organizadas según la vertiente teórica predominante y destaca sus contribuciones principales.

Siguiendo con el análisis de los artículos incorporados al dominio «Territorios y procesos de desarrollo", realizamos también un estudio de las referencias bibliográficas que daban soporte a los mismos. El objetivo de este abordaje era identificar a los autores clásicos de la teoría de resiliencia independientemente del enfoque utilizado, ya que se consideró que estos adquirían importancia a los fines de comprender la evolución del concepto. En esta aproximación, se destacan los autores consignados anteriormente en el análisis bibliométrico, como, por ejemplo: B. H. Walker y N. Adger, además de otros como C. S. Holling, C. Folke, F. Berkes y L. H. Gunderson. Carl Folke y C. S. Holling son los investigadores más citados habitualmente en los artículos en cuestión. Esto no sorprende, pues ya Janssen et al. (2006) habían identificado al artículo del profesor Folke de la Universidad de Estocolmo (Suecia) como aquel con el mayor número de citas en publicaciones sobre resiliencia.

Carl Folke ejerce como director del Stockholm Resilience Center y es miembro del Comité Ejecutivo y del Consejo de Ciencia de la Resilience Alliance. Cuenta con una vasta experiencia en colaborar con científicos de las ciencias naturales y sociales; además, ha trabajado la dimensión social y económica de la gestión del ecosistema y ha propuesto medidas proactivas para evitar la pérdida de resiliencia.

Sin embargo, Crawford S. Holling, actualmente profesor emérito de la Universidad de Florida y uno de los fundadores del grupo Resilience Alliance, es aún uno de los autores más citados. Su contribución de mayor relevancia aparece en el artículo publicado en 1973 y titulado «Resilience and stability of ecological systems», donde introduce el concepto de resiliencia en el campo de la ecología. Este texto, con el tiempo, acabó por impactar no solamente en la ecología, sino también en otras ciencias naturales y sociales. 
Tabla 5. Referencias bibliográficas clasificadas según la vertiente teórica predominante y sus contribuciones principales

\begin{tabular}{lll}
\hline Referencia & $\begin{array}{l}\text { Vertiente teórica } \\
\text { predominante }\end{array}$ & Aspectos generales y contribuciones principales \\
\hline $\begin{array}{l}\text { Adger et al. (2002) } \\
\text { y Marshall et al. }\end{array}$ & Resiliencia social & $\begin{array}{l}\text { Estos artículos están entre los más antiguos después de Adger (2000) y tienen, en este último, } \\
\text { una fuerte inspiración conceptual. Discuten la resiliencia de sistemas socioecológicos a partir } \\
\text { (2007) }\end{array}$ \\
$\begin{array}{l}\text { del concepto de resiliencia social y presentan como contribución principal el esfuerzo en } \\
\text { aplicar este concepto en estudios de caso. }\end{array}$
\end{tabular}

Pickett et al. (2004) Resiliencia urbana El concepto de resiliencia urbana se vincula con la planificación de las poblaciones. En este caso, la resiliencia está fuertemente influida por percepciones ecológicas. La metáfora de «ciudades resilientes» es una herramienta que puede sintetizar las relaciones entre planificación urbana, ciencias ecológicas y sociales. La principal contribución es conceptual y metodológica. El gran peso de la idea de resiliencia ecológica hace que este trabajo no se vincule directamente con el campo del desarrollo regional. Dicho problema es muy común en las publicaciones de este período.

Ernstson et al. Resiliencia urbana Buscan contribuir a superar la ausencia de teorías que expandan el uso de la idea de resi(2010) liencia urbana a los estudios de sistemas urbanos. El trabajo se sustenta en un estudio de caso de tres ciudades. De la misma forma que Pickett et al. (2004), aparece como un artículo conceptual y metodológico, con contribuciones más adecuadas ¿que el anterior? al estudio de la resiliencia urbana.

\begin{tabular}{lll}
\hline Magis (2010) & Resiliencia & El concepto de resiliencia comunitaria defiende que las comunidades son capaces de adaptar- \\
& comunitaria & $\begin{array}{l}\text { se transformarse mediante los procesos de crisis, y propone ocho dimensiones que facilitan } \\
\text { medir la resiliencia de una comunidad. Sus contribuciones principales son metodológicas. }\end{array}$
\end{tabular}

Berkes y Ross Resiliencia $\quad$ Tal como sugiere Margis (2010), los autores adoptan el concepto de resiliencia comunitaria. (2013) comunitaria Su distinción reside en el hecho de que la comunidad se sitúa en el centro de estudio de la resiliencia a través de dos vertientes: la de los sistemas socioecológicos y la de la psicología y la salud mental. Según los autores citados, la unión de estas dos vertientes podría ofrecer informaciones sobre la resiliencia que no estarían disponibles sin este uso integrado.

Walker et al. (2009) Resiliencia regional Aplican la teoría de la resiliencia. Usan como base la idea de ciclos adaptativos para evaluar la sustentabilidad de una región en el sudeste de Australia. Es un artículo con interesantes contribuciones metodológicas.

Wardekker et al. Resiliencia regional Proponen aplicar el concepto de resiliencia (como una aportación adaptada) al análisis de los (2010) cambios climáticos. Aplican seis principios de resiliencia en un estudio de caso en Holanda. Aporta interesantes contribuciones metodológicas de difícil aplicación en estudios orientados al desarrollo regional.

Wilson (2010) Resiliencia rural Contribuye al debate emergente de la resiliencia en comunidades rurales. Para ello, se centra en la necesidad de construir un nuevo concepto de multifuncionalidad, integrado en los debates actuales de resiliencia. Como tal, aporta contribuciones conceptuales significativas, especialmente para los estudios orientados a la resiliencia de regiones rurales.

\begin{tabular}{|c|c|c|}
\hline $\begin{array}{l}\text { Christopherson et } \\
\text { al. (2010), Bristow } \\
\text { (2010), Hassink } \\
\text { (2010), Hudson } \\
\text { (2009) y Simmie }\end{array}$ & Resiliencia regional & $\begin{array}{l}\text { Estos artículos analizan y discuten la evolución del concepto de resiliencia y su potencial para } \\
\text { explicar el desarrollo regional. Exploran ideas como las de adaptación y de transformación y/o } \\
\text { el modelo de ciclos adaptativos como un camino adecuado para analizar la resiliencia. Son de } \\
\text { gran utilidad para comprender las potencialidades y las fragilidades de este concepto cuando } \\
\text { se aplica en el contexto del desarrollo regional. }\end{array}$ \\
\hline
\end{tabular}
y Martin (2010)

Cote y Nightingale Resiliencia social / Los autores señalan problemas epistemológicos y normativos que resultan de la migración (2012) Resiliencia del concepto de resiliencia desde los análisis de los sistemas ecológicos hacia los sistemas sociales. Para enfrentar estos problemas, proponen el uso de una «epistemología compartida» que permita mediar la relación entre los campos del conocimiento. Este trabajo procura lanzar luz sobre una serie de inconsistencias en el modo como las investigaciones sobre los sistemas socioecológicos se involucran en cuestiones normativas.

Fuente: elaboración propia. 


\section{Conclusiones}

Los análisis de las publicaciones aquí presentadas y discutidas han intentado ofrecer orientaciones respecto a los caminos que podrían seguir los interesados en discutir el concepto de resiliencia aplicado al desarrollo regional.

La revisión de la literatura demuestra que el proceso de migración del concepto hacia las ciencias sociales y económicas es complejo y se contrapone a la premisa del equilibrio - común a las ciencias biológicas y las ingenierías-, en la medida en que la idea de resiliencia reconoce la hipótesis de que debe existir una capacidad de adaptación en los territorios, especialmente en las regiones bajo contextos desfavorables.

El registro y la medición de la producción científica en torno al concepto y su evolución reciente ofrecen pistas importantes para la organización del análisis del estado de la cuestión. La división de la literatura en dominios lingüísticos ha mostrado que se trata de una herramienta útil para esta organización. A su vez, posibilitó el estudio del concepto de resiliencia en el ámbito temático que interesa específicamente a este artículo. Asimismo, la selección de los términos de búsqueda permitió que se pudieran comparar las producciones según la escala geográfica y el objeto de estudio.

Se observó la tendencia al fraccionamiento de la escala espacial de los estudios, inicialmente muy apegada a la unidad «región». Tal fragmentación viene induciendo a cambios sutiles, pero importantes, en la naturaleza del objeto investigado. Por ejemplo, es cada vez más común el uso de los términos community resilience, resilient cities, urban resilience y rural resilience.

En el conjunto de las publicaciones, destaca la revista Cambridge Journal of Regions Economy and Society, y entre los autores: Adger (2000), Norris et al. (2008), Christopherson et al. (2010), Bristow (2010), Hassink (2010), Hudson (2009) y Simmie y Martin (2010), los cuales produjeron artículos que son una referencia en el estudio de las relaciones entre la resiliencia y el desarrollo regional.

Es evidente la hegemonía de la producción en lengua inglesa y de las revistas publicadas en Estados Unidos y en el Reino Unido en el desarrollo del concepto de resiliencia. De hecho, ambos países se constituyeron en los centros de promoción y difusión del debate.

Finalmente, en términos generales, se puede afirmar que el análisis bibliométrico permitió el enriquecimiento y la cualificación del estudio de la evolución del planteamiento conceptual relacionado con el desarrollo regional. Esta metodología ha contribuido a facilitar un mayor conocimiento, con amplitud y profundidad, del proceso de construcción de un nuevo aporte conceptual en los estudios que tienen por objetivo el desarrollo regional. 


\section{Agradecimientos}

El primer autor agradece a CAPES la concesión de la beca de doctorado sándwich $^{2}$ en el Departamento de Geografía de la Universitat Autònoma de Barcelona (UAB), que ha contribuido a hacer posible este trabajo.

\section{Referencias bibliográficas}

Adger, N. (2000). «Social and ecological resilience: Are they related?». Progress in Human Geography [en línea], 24 (3), 347-364. $<$ http://dx.doi.org/10.1191/030913200701540465>

Adger, N. et al. (2002). «Migration, remittances, livelihood trajectories, and social resilience». Ambio [en línea], 31 (4), 358-366. $<$ http://dx.doi.org/10.1579/0044-7447-31.4.358>

Berkes, F. y Ross, H. (2013). "Community resilience: toward an integrated approach». Society and Natural Resources, 26, 5-20.

Bristow, G. (2010). «Resilient regions: re-'place'ing regional competitiveness». Cambridge Journal of Regions, Economy and Society [en línea], 3, 153-167. <http://dx.doi.org/10.1093/cjres/rsp030>.

Cabell, J. y Oelofse, M. (2012). «An indicator framework for assessing agroecosystem resilience». Ecology and Society [en línea], 17 (1), 18. $<$ http://dx.doi.org/10.5751/es-04666-170118>.

Campanella, T. J. (2006). «Urban resilience and the recovery of New Orleans». Journal of the American Planning Association [en línea], 72 (2), 141-146. $<$ http://dx.doi.org/10.1080/01944360608976734>.

Christopherson, S.; Michieb, J. y Tylerc, P. (2010). «Regional resilience: Theoretical and empirical Perspectives». Cambridge Journal of Regions, Economy and Society [en línea], 3, 3-10. $<$ http://dx.doi.org/10.1093/cjres/rsq004>.

Cote, N. y Nightingale, A. J. (2012). «Resilience thinking meets social theory: situating social change in socio-ecological systems (SES) research». Progress in Human Geography, 36 (4), 475-489.

DARNHOFER, I. (2014). «Resilience and why it matters for farm management». European Review of Agricultural Economics [en línea]. $<\mathrm{http} / /$ dx.doi.org/10.1093/erae/jbu012>

Darnhofer, I.; Fairweather, J. y Moller, H. (2010). «Assessing a farm's sustainability: Insights from resilience thinking». International Journal of Agricultural Sustainability [en línea], 8, 186-198. $<$ http://dx.doi.org/10.3763/ijas.2010.0480>

Ernstson, H. et al. (2010). "Urban Transitions: On Urban Resilience and Human-Dominated Ecosystems». Ambio [en línea], 39 (8), 531-545. $<$ http://dx.doi.org/10.1007/s13280-010-0081-9>

2. Ayuda del Gobierno brasileño a los estudiantes de doctorado para que puedan realizar una estancia de un año en otro país. El director de tesis en la Universidade Federal de Santa Catarina (UFSC) es el Dr. Clecio Azevedo da Silva, y su tutor, el Dr. Antoni F. Tulla (UAB). 
Franklin, A.; Newton, J. y McEntee, J. C. (2011). «Moving beyond the alternative: Sustainable communities, rural resilience and the mainstreaming of local food». Local Environment [en línea], 16, 771-788. <http://dx.doi.org/10.1080/13549839.2011.574685>

Glover, J. (2012). "Rural resilience through continued learning and innovation». Local Economy [en línea], 27 (4), 355-372. <http://dx.doi.org/10.1177/0269094212437833>

Hassink, R. (2010). «Regional resilience: A promising concept to explain differences in regional economic adaptability?». Cambridge Journal of Regions, Economy and Society [en línea], 3, 45-58. <http://dx.doi.org/10.1093/cjres/rsp033>

Hill, E. W.; Wial, H. y Wolman, H. (2008). «Exploring Regional Economic Resilience». Working Paper 2008-04. Institute of Urban and Regional Development, Berkeley.

Hopkins, R. (2008). The Transition Handbook: From Oil Dependency to Local Resilience. Chelsea: Green Books.

Hudson, R. (2009). "Resilient regions in an uncertain world: Wishful thinking or a practical reality?». Cambridge Journal of Regions, Economy and Society [en línea], 3, 11-25. <http://dx.doi.org/10.1093/cjres/rsp026>

Janssen, M. A. et al. (2006). «An update on the scholarly networks on resilience, vulnerability, and adaptation within the human dimensions of global environmental change». Ecology and Society, 12 (2).

KING, C. A. (2008). «Community resilience and contemporary agri-ecological systems: Reconnecting people and food, and people with people». Systems Research and Behavioral Science [en línea], 25, 111-124. <http://dx.doi.org/10.1002/sres.854>

Magis, K. (2010). "Community Resilience: An Indicator of Social Sustainability». Society \& Natural Resources: An International Journal [en línea], 23(5), 401-416. <http://dx.doi.org/10.1080/08941920903305674>

Marshall, N. A. et al. (2007). «How Resource Dependency Can Influence Social Resilience within a Primary Resource Industry». Rural Sociology, 72 (3), 359-390. <http://dx.doi.org/10.1526/003601107781799254>

Méndez, R. (2012). "Ciudades y metáforas: Sobre el concepto de resiliencia urbana». Ciudad y Territorio: Estudios Territoriales, 172, 215-231.

- (2013). «Estrategias de innovación para el desarrollo y la resiliencia de ciudades medias». Documents d'Anàlisi Geogràfica, 59 (3), 481-499.

Norris, F. H. et al. (2008). "Community Resilience as a Metaphor, Theory, Set of Capacities, and Strategy for Disaster Readiness». American Journal of Community Psychology [en línea], 4, 127-150. <http://dx.doi.org/10.1007/s10464-007-9156-6>

Paniagua, A. (2013). "Farmers in remote rural areas: The worth of permanence in the place». Land Use Policy [en línea], 35, 1-7. <http://dx.doi.org/10.1016/j.landusepol.2013.04.017>

Pendall, R.; Foster, K. A. y Cowell, M. (2009). «Resilience and regions: Building understanding of the metaphor». Cambridge Journal of Regions, Economy and Society [en línea], 3, 71-84. <http://dx.doi.org/10.1093/cjres/rsp028> 
Pickett, S. T. A.; Cadenasso, M. L. y Grove, J. M. (2004). «Resilient cities: Meaning, models, and metaphor for integrating the ecological, socio-economic, and planning realms». Landscape and Urban Planning [en línea], 69, 369-384. <http://dx.doi.org/10.1016/j.landurbplan.2003.10.035>

Pike, A.; Dawley, S. y Tomaney, J. (2010). «Resilience, adaptation and adaptability». Cambridge Journal of Regions, Economy and Society [en línea], 3, 59-70. <http://dx.doi.org/10.1093/cjres/rsq001>

Polèse, M. (2010). «The resilient city: On the determinants of successful urban economies». Working Paper, 2010-03. Montreal. INRS, University of Quebec.

SAntos, F. T. (2009). "Territórios resilientes enquanto orientação de planeamento». Prospectiva e Planeamento, 16.

Sсотт, M. (2013). «Resilience: A conceptual lens for rural studies?». Geography Compass, 7/9, 597-610. <http://dx.doi.org/10.1111/gec3.12066>

Simmie, J. y Martin, R. L. (2010). "The economic resilience of regions: Towards an evolutionary approach». Cambridge Journal of Regions, Economy and Society [en línea], 3, 27-43. <http://dx.doi.org/10.1093/cjres/rsp029>

WALKer, B. et al. (2009). «Resilience, adaptability, and transformability in the Goulburn-Broken Catchment, Australia». Ecology and Society, 14 (1), 12.

WARDEKKer, J. A. et al. (2010). "Operationalising a resilience approach to adapting an urban delta to uncertain climate changes». Technological Forecasting \& Social Change [en línea], 77, 987-998. <http://dx.doi.org/10.1016/j.techfore.2009.11.005>

Wilson, G. (2010). "Multifunctional "quality" and rural community resilience». Transactions of the Institute of British Geographers [en línea], 35, 364-381. <http://dx.doi.org/10.1111/j.1475-5661.2010.00391.x>

Wilson, S. G. et al. (2013). «Separating adaptive maintenance (resilience) and transformative capacity of social-ecological systems». Ecology and Society [en línea], 18 (1), 22 . <http://dx.doi.org/10.5751/ES-05100-180122> 